\title{
OS EFEITOS DAS ALTERAÇÕES COMPORTAMENTAIS DAS VÍTIMAS DE TRAUMA CRÂNIO-ENCEFÁLICO PARA O CUIDADOR FAMILIAR ${ }^{1}$
}

Edilene Curvelo Hora ${ }^{2}$

Regina Márcia Cardoso de Sousa ${ }^{3}$

Hora EC, Sousa RMC. Os efeitos das alterações comportamentais das vítimas de trauma crânio-encefálico para o cuidador familiar. Rev Latino-am Enfermagem 2005 janeiro-fevereiro; 13(1):93-8.

Oestudo teve como objetivos identificar alterações na intensidade que os comportamentos negativos das vítimas de Trauma Craniencefálico (TCE) afetavam o cuidador familiar principal, comparando o período anterior ao posterior ao trauma e verificar a relação entre intensidade dessas alterações e tempo decorrido após o evento traumático. Incluiu 50 cuidadores de vítimas com diferentes níveis de dependência após TCE. Os efeitos dos comportamentos da vítima para o cuidador foram mensurados por meio da escala Likert, tendo em vista onze comportamentos negativos citados em literatura. Na visão do cuidador, a vítima ficou mais agressiva, ansiosa, dependente, deprimida, irritada, esquecida, com temperamento mais explosivo, após o trauma, além de mais egocêntrica, impulsiva, com maior inadequação social e oscilação de humor. Os seis primeiros comportamentos citados eram os que afetaram mais negativamente o cuidador. Não houve relação entre o tempo decorrido e os efeitos das alterações comportamentais.

DESCRITORES: traumatismos cerebrais; cuidadores; enfermagem familiar; comportamento

\section{EFFECT OF THE BEHAVIORAL ALTERATIONS OF VICTIMS OF TRAUMATIC BRAIN INJURY FOR THE FAMILY CAREGIVER}

This study aimed to identify alterations in the intensity at which the negative behaviors of the victims of traumatic brain injury (TBI) affect the main family caregiver comparing the periods before and after the trauma and to verify the relation between the intensity of these alterations and time passed after the traumatic event. Participants were 50 caregivers of victims with different levels of dependence after TBI. The effect of the victim's behaviors on the caregiver was measured by means of a Likert scale, in view of eleven negative behaviors cited in literature. According to the caregiver, the victim was more aggressive, anxious, dependent, depressed, irritated, and forgetful after the trauma, with a more explosive temperament, more self-centered, impulsive, with greater social inadequacy and mood oscillation. The first six cited behaviors were the ones that affected the caregiver more negatively. No relation was found between the passed time and the effect of the behavioral alterations

DESCRIPTORS: brain injuries; caregivers; family nursing; behavior

\section{LOS EFECTOS DE LAS ALTERACIONES COMPORTAMENTALES DE LAS VICTIMAS DE TRAUMA CRÁNEO ENCEFÁLICO PARA EL CUIDADOR FAMILIAR}

El estudio tiene como objetivos identificar las alteraciones en la intensidad con que los comportamientos negativos de las victimas de Trauma Cráneo-Encefálico (TCE) inciden sobre el cuidador familiar principal, comparando los periodos anterior y posterior al trauma, y verificar la relación entre la intensidad de esas alteraciones y el tiempo transcurrido después del evento traumático. Los participantes son 50 casos de cuidadores de victimas con diferentes niveles de dependencia tras TCE. Los efectos de los comportamientos de la victima para con el cuidador fueron medidos por medio de una escala Likert, teniéndose en cuenta once comportamientos negativos mencionados en la literatura. En la visión del cuidador, la victima después del trauma está más agresiva, ansiosa, dependiente, deprimida, irritada, olvidadiza, con temperamento más explosivo, además de más egocentrista, impulsiva, con mayor inadecuación social y variación de humor. Los seis primeros comportamientos mencionados fueron los que más incidieron negativamente sobre el cuidador. No se encontró relación entre el tiempo transcurrido y los efectos de las alteraciones comportamentales.

DESCRIPTORES: traumatismos cerebrales; cuidadores; enfermería de la familia; conducta

\footnotetext{
${ }^{1}$ Trabalho extraído da dissertação de mestrado apresentada à Escola de Enfermagem da Universidade de São Paulo, a pesquisa contou com auxílio da FAPESP, Processo no 01/09645-7; ${ }^{2}$ Enfermeira, Doutoranda em Enfermagem, Professor Assistente da Universidade Federal de Sergipe, e-mail: lenehora@uol.com.br; ${ }^{3}$ Enfermeira, Professor Associado da Escola de Enfermagem da Universidade de São Paulo, e-mail: vian@usp.br
} 


\section{INTRODUÇÃO}

No Brasil, os acidentes e a violência configuram um problema de saúde pública de grande magnitude e transcendência, com forte impacto na morbidade e na mortalidade da população. No conjunto das lesões decorrentes das causas externas, o Trauma Craniencefálico (TCE) destaca-se em termos de magnitude tanto entre mortos quanto feridos, sendo uma das lesões mais freqüentes ${ }^{(1)}$.

OTCE é conceituado como qualquer agressão que acarreta lesão anatômica ou comprometimento funcional do couro cabeludo, crânio, meninges ou encéfalo ${ }^{(2)} \mathrm{e}$, de um modo geral, encontra-se dividido, segundo sua intensidade, em grave, moderado e leve. É considerado como processo dinâmico, já que as conseqüências de seu quadro patológico podem persistir e progredir com o passar do tempo ${ }^{(3)}$.

As vítimas que sobrevivem ao TCE podem apresentar deficiências e incapacidades que são temporárias ou permanentes, interferindo na capacidade do indivíduo de desempenhar suas funções ${ }^{(4)}$.

As incapacidades resultantes do TCE podem ser divididas em três categorias físicas, cognitivas e comportamentais/emocionais. As incapacidades físicas são diversificadas, podendo ser visuais, motoras, entre outras; as incapacidades cognitivas, freqüentemente, incluem diminuição da memória, dificuldades de aprendizagem, entre outras; e as comportamentais/emocionais são a perda de autoconfiança, comportamento infantil, motivação diminuída, e mais comumente, irritabilidade e agressão ${ }^{(5)}$.

Recente revisão bibliográfica, que descreve os fatores relacionados com o prognóstico das vítimas de TCE, apontou que as alterações neuropsicológicas pós-traumáticas constituem um dos principais fatores que determinam o futuro dessas pessoas, pois condiciona, de forma notável, tanto o grau de independência funcional alcançado e retorno ao trabalho, como também o estabelecimento de relações familiares e sociais satisfatórias ${ }^{(6)}$.

Corroborando com essas observações, as pesquisas que descrevem as conseqüências negativas vivenciadas pelos cuidadores em decorrência ao cuidado das vítimas de TCE mostram que suas causas são mais determinadas pelas mudanças comportamentais, emocionais, sociais e cognitivas desenvolvidas pelas vítimas de TCE que pelas mudanças associadas com a incapacidade física ${ }^{(7-9)}$.

Diante de tais constatações, resolveu-se estudar os efeitos dos comportamentos negativos das vítimas de TCE para a família, na pessoa do cuidador familiar principal, considerando que ele tem a responsabilidade maior e permanente da vítima sob seu cuidado e, dessa forma, poderá também retratar e sofrer as conseqüências do evento traumático.

De acordo com esse propósito, e tendo em vista que o tempo decorrido após o trauma pode ser variável importante nesse tipo de estudo, foram estabelecidos os seguintes objetivos para o presente estudo: identificar possíveis diferenças na intensidade que os comportamentos das vítimas afetavam o cuidador antes do trauma e 0 afetam atualmente ( $\geq$ seis meses após o trauma) e verificar a relação entre a intensidade das alterações observadas e tempo decorrido após o trauma.

\section{CASUÍSTICA E MÉTODO}

Este estudo caracteriza-se como descritivo, exploratório, de campo, prospectivo e com abordagem quantitativa. Desenvolvido no Ambulatório de Trauma de Crânio do Hospital das Clínicas da Faculdade de Medicina da Universidade de São Paulo (HCFMUSP), no período de 27 de junho a 5 de dezembro de 2001, após aprovação em Comissão de Ética e Pesquisa.

A casuística em estudo foi constituída de 50 cuidadores de vítimas de TCE que atenderam aos seguintes critérios de inclusão: comparecer ao Ambulatório, em decorrência do acompanhamento da vítima; ter idade igual ou superior a 18 anos e cuidar de vítimas de TCE na faixa etária entre 12 e 60 anos; ser cuidador familiar principal da vítima de TCE que sofreu evento traumático há pelo menos seis meses, período que o quadro da vítima já se encontra estável, segundo pesquisas que analisaram o processo de recuperação após TCE ${ }^{(10-11)}$. A participação do cuidador no presente estudo foi vinculada ao seu consentimento verbal e escrito.

Foi realizada entrevista estruturada, individual e única, por meio da aplicação de um formulário que compreendeu os dados para caracterização do cuidador e a avaliação dos efeitos dos comportamentos exibidos pelas vítimas de TCE antes do trauma e na época da entrevista (Figura 1). Essa avaliação, adaptada da elaborada por pesquisadores da Universidade de Indianápolis nos Estados Unidos $^{(12)}$, contém uma relação dos comportamentos das vítimas de TCE descritos na literatura e indicados como os mais freqüentes incômodos aos cuidadores após o trauma. A aplicação dos questionários contidos nessa avaliação, em conjunto com a escala Likert com cinco categorias, permitiu registrar a intensidade que os comportamentos negativos afetavam cuidador.

Para facilitar o uso da referida escala criou-se cinco expressões faciais relacionadas a sentimentos que variam de nenhum efeito (um) a máximo efeito (cinco), ilustradas na Figura 1 e apresentadas ao cuidador no momento da entrevista.

O questionamento de cada comportamento foi feito separadamente, indagando-se seqüencialmente sobre quanto os comportamentos afetavam o familiar antes do trauma e na época da entrevista. Para auxiliar o cuidador a identificar os comportamentos 
listados foram utilizadas as descrições e definições presentes na Figura 1.

A listagem dos comportamentos com suas respectivas definições seguem na íntegra o conteúdo apresentado nos instrumentos originais descritos em inglês ${ }^{(12)}$. Para manter a fidedignidade, a tradução, após ser realizada pelas pesquisadoras e revisada por professora da língua inglesa, foi analisada por duas enfermeiras, doutoras em enfermagem, com domínio da língua inglesa que avaliaram a clareza e propriedade da tradução realizada.

As fontes de dados das informações das vítimas, apresentadas nos resultados deste estudo, foram os prontuários e formulários de uma pesquisa complementar realizada no local de estudo ${ }^{(13)}$.

Os dados foram armazenados em um banco de dados computadorizado, construído com o programa SPSS 8.0. Para a comparação dos resultados relacionados ao comportamento das vítimas antes do trauma e atualmente ( $\geq$ seis meses após o trauma), trabalhouse com a média dos escores das duas informações do cuidador (antes do trauma e seis meses ou mais após). Sendo assim, foram aplicados testes pareados para comparação desses resultados e como, nesse caso, a suposição de normalidade dos dados não estava satisfeita (tem-se dados ordinais que variam de 1 a 5), foi empregado o teste não-paramétrico de Wilcoxon.

$\mathrm{Na}$ comparação entre os diversos comportamentos, trabalhou-se com as diferenças dos escores atribuídos individualmente pelo cuidador após e antes do trauma, ou seja, valores positivos dessa diferença indicavam que houve aumento na escala após o trauma e valores negativos dessa diferença mostravam diminuição da escala após o trauma. A comparação dessas diferenças foi feita por medidas descritivas e pelo teste não-paramétrico de Friedman.

A associação entre o tempo de trauma da vítima (menor ou igual a três anos e maior que três anos) e a diferença na pontuação na Escala Likert de cada um dos comportamentos, foi realizada por meio das medidas descritivas e do teste não-paramétrico de Mann-Whitney. O nível de significância de $5 \%$ foi estabelecido para todas as análises realizadas.

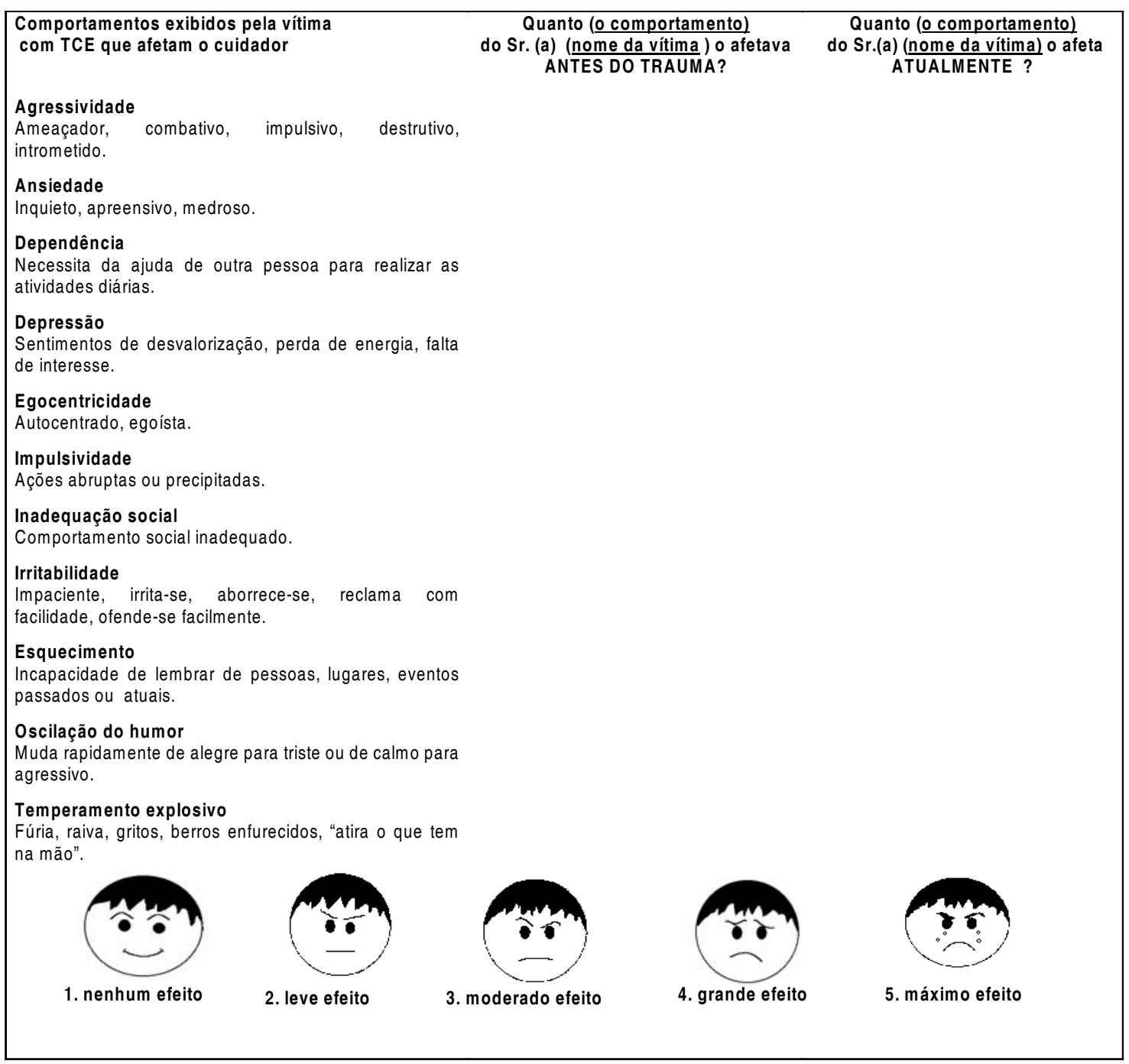

${ }^{*}$ Adaptada de pesquisadores da Universidade de Indianápolis nos Estados Unidos ${ }^{(9)}$

Figura 1 - Avaliação dos efeitos dos comportamentos da vítima de TCE para o cuidador 


\section{RESULTADOS}

O estudo incluiu 50 cuidadores com idade média de 44,90 anos e desvio padrão de 13,19 , sendo $88 \%$ do sexo feminino, $68 \%$ solteiros, $58 \%$ católicos, $60 \%$ com ensino fundamental incompleto e $64 \%$ exerciam ocupação remunerada antes do evento traumático. A metade dos cuidadores em estudo era mãe da vítima, 22\% esposo(a) e os demais, irmão(ã), pai, tio(a) ou filho(a).

Esses cuidadores eram os responsáveis principais pelo cuidado de 50 vítimas de TCE, com idade média de 30,60 anos e desvio padrão de 12,08 , sendo $90 \%$ do sexo masculino, $60 \%$ casados ou amasiados, em $74 \%$ a causa externa foi o acidente de transporte. 0 tempo médio decorrido do trauma na época da entrevista foi 4,42 anos com desvio padrão igual a 4,82, variando entre 6 meses e 18 anos, sendo que $40 \%$ tinham mais que 3 anos. Quanto à condição da vítima após TCE, a maioria, 56\% eram incapazes e dependentes de um cuidador; 14\%, embora apresentassem alguma incapacidade, eram independentes e 30\% não tinham incapacidades após TCE, entretanto, podiam apresentar alguma deficiência física ou mental.

A análise descritiva dos resultados da escala Likert em relação à intensidade que os comportamentos da vítima afetavam o cuidador antes e seis meses, ou mais, após o trauma está apresentada nos dados das Tabelas 1 e 2 .

Tabela 1 - Média e desvio padrão dos resultados da escala Likert em relação aos comportamentos, antes do trauma e atualmente ( $\geq 6$ meses após o trauma). IC-HCFMUSP, 2001

\begin{tabular}{lcccc}
\hline COMPORTAMENTOS & \multicolumn{2}{c}{ ANTES DO TRAUMA } & \multicolumn{2}{c}{ ATUALMENTE } \\
& Média & Desvio Padrão & Média & Desvio Padrão \\
\hline Agressividade & 1,20 & 0,70 & 3,28 & 1,60 \\
Ansiedade & 1,08 & 0,34 & 3,30 & 1,43 \\
Dependência & 1,06 & 0,31 & 3,34 & 1,62 \\
Depressão & 1,14 & 0,64 & 3,30 & 1,47 \\
Egocentricidade & 1,08 & 0,34 & 2,36 & 1,65 \\
Impulsividade & 1,14 & 0,61 & 2,78 & 1,52 \\
Inadequação social & 1,02 & 0,14 & 2,18 & 1,64 \\
Irritabilidade & 1,20 & 0,70 & 3,40 & 1,53 \\
Esquecimento & 1,00 & 0,00 & 3,44 & 1,49 \\
Oscilação do humor & 1,10 & 0,42 & 2,80 & 1,50 \\
Temperamento explosivo & 1,08 & 0,40 & 3,14 & 1,75 \\
\hline
\end{tabular}

Tabela 2 - Mediana e valores mínimo e máximo dos resultados da escala Likert, em relação aos comportamentos, antes do trauma e atualmente ( $\geq 6$ meses após o trauma). IC-HCFMUSP, 2001

\begin{tabular}{lcccccc}
\hline COMPORTAMENTOS & \multicolumn{3}{c}{ ANTES DO TRAUMA } & \multicolumn{3}{c}{ ATUALMENTE } \\
& Mínimo & Mediana & Máximo & Mínimo & Mediana & Máximo \\
\hline Agressividade & 1 & 1 & 5 & 1 & 3 & 5 \\
Ansiedade & 1 & 1 & 3 & 1 & 3 & 5 \\
Dependência & 1 & 1 & 3 & 1 & 4 & 5 \\
Depressão & 1 & 1 & 5 & 1 & 4 & 5 \\
Egocentricidade & 1 & 1 & 3 & 1 & 1 & 5 \\
Impulsividade & 1 & 1 & 5 & 1 & 3 & 5 \\
Inadequação social & 1 & 1 & 2 & 1 & 1 & 5 \\
Irritabilidade & 1 & 1 & 5 & 1 & 4 & 5 \\
Esquecimento & 1 & 1 & 1 & 1 & 4 & 5 \\
Oscilação do humor & 1 & 1 & 3 & 1 & 3 & 5 \\
Temperamento explosivo & 1 & 1 & 3 & 1 & 4 & 5 \\
\hline
\end{tabular}

Observa-se que a tendência dos escores de todos os comportamentos foi aumentar após o trauma (aumento das médias e medianas). A pontuação média da escala Likert em relação aos comportamentos variou de 1,00 a 1,20 antes do trauma e de 2,18 a 3,44 após o trauma. Evidencia-se que antes do trauma houve pequena variabilidade dos dados (baixo desvio padrão) pela grande concentração das respostas no escore um (nenhum efeito), diferenciando-se do período após o trauma em que houve maior variabilidade dos dados (maior desvio padrão) e grande concentração das respostas nos escores mais altos (moderado e grande efeito). Fato que pode ser constatado nos valores das medianas que antes do trauma mantinham-se em 1 (nenhum efeito), em todos os comportamentos, e passaram para 3 (moderado efeito) ou 4 (grande efeito), exceto nos dados relativos à egocentricidade e inadequação social. Pela média e desvio padrão, observa-se que o comportamento da vítima 'esquecimento' não afetava nenhum dos cuidadores no período que antecedeu o trauma.

Verificou-se também na análise dos dados que, em relação à inadequação social, egocentricidade, oscilação do humor e esquecimento, as respostas sempre indicaram manutenção ou aumento da intensidade que esses comportamentos afetavam o cuidador após o TCE. Nos demais, agressividade, ansiedade, depressão, impulsividade, irritabilidade, temperamento explosivo e dependência, alguns cuidadores também indicaram menor efeito após o evento traumático.

Com a aplicação do teste de Wilcoxon, pode-se relatar que as diferenças nas médias de todos os comportamentos estudados, antes do trauma e após, foram diferentes de zero $(p=0,00)$, sendo consideradas, portanto, significativas.

Ainda estudando os efeitos das mudanças dos comportamentos das vítimas para o cuidador, uma análise descritiva dos resultados das diferenças dos escores atribuídos para cada um dos cuidadores, após o trauma e antes, aparece nos dados da Tabela 3, que apresenta, em ordem crescente, a média dessas diferenças acompanhada das demais estatísticas descritivas. Nota-se, como esperado pelas análises anteriores, que as médias das diferenças são positivas para todos os comportamentos, ou seja, em média houve aumento dos escores após o trauma, no entanto, esse aumento médio variou de 1,16 a 2,44. Também, verifica-se que alguns comportamentos como inadequação social e egocentricidade apresentaram resultados descritivos bastante similares e, por outro lado, comportamentos como esquecimento e egocentricidade ou impulsividade foram bem diferentes. Em média, o comportamento que mais alterou na percepção do cuidador foi o esquecimento. 
Tabela 3 - Estatísticas descritivas das diferenças entre os escores dos comportamentos após e antes do trauma. IC-HCFMUSP, 2001

\begin{tabular}{lccccc}
\hline COMPORTAMENTOS & \multicolumn{5}{c}{ ESTATISTICAS DESCRITIVAS } \\
& Média & Desvio Padrão & Mínimo & Mediana & Máximo \\
\hline Inadequação social & 1,16 & 1,63 & 0,00 & 0,00 & 4,00 \\
Egocentricidade & 1,28 & 1,68 & 0,00 & 0,00 & 4,00 \\
Impulsividade & 1,64 & 1,69 & $-3,00$ & 1,00 & 4,00 \\
Oscilação do humor & 1,70 & 1,52 & 0,00 & 2,00 & 4,00 \\
Temperamento explosivo & 2,06 & 1,82 & $-2,00$ & 2,50 & 4,00 \\
Agressividade & 2,08 & 1,85 & $-3,00$ & 2,00 & 4,00 \\
Depressão & 2,16 & 1,50 & $-1,00$ & 3,00 & 4,00 \\
Irritabilidade & 2,20 & 1,68 & $-2,00$ & 3,00 & 4,00 \\
Ansiedade & 2,22 & 1,54 & $-1,00$ & 2,00 & 4,00 \\
Dependência & 2,28 & 1,71 & $-1,00$ & 3,00 & 4,00 \\
Esquecimento & 2,44 & 1,49 & 0,00 & 3,00 & 4,00 \\
\hline
\end{tabular}

Considera-se importante salientar que o interesse principal para essa outra forma de análise dos dados foi verificar se houve alguma diferença significativa no aumento desses comportamentos. Pelo teste não-paramétrico de Friedman, rejeitou-se a hipótese de que as diferenças de todos os comportamentos fossem iguais $(p=0,00)$, fazendo-se a comparação de cada dois comportamentos, de uma forma geral, dois grupos de comportamentos com diferenças médias iguais foram formados, sendo que o primeiro foi em média menor que 0 segundo.

1ำ grupo: inadequação social, egocentricidade, impulsividade e oscilação de humor;

2ํrupo: temperamento explosivo, agressividade, depressão, irritabilidade, ansiedade, dependência e esquecimento.

Além das análises anteriores, e considerando a distribuição bastante assimétrica de tempo do trauma na época da entrevista, verificou-se a associação entre o tempo de trauma da vítima e as diferenças entre os escores após e antes do trauma. Para cada um dos comportamentos em estudo, foi feita uma comparação das médias dessas diferenças em vítimas com tempo de trauma menor ou igual a três anos, em recuperação a curto e médio prazos $(n=30)$ e maior que três anos, em recuperação a longo prazo $(n=20)$. A análise descritiva desses resultados está apresentada nos dados da Tabela 4, onde se observou que as diferenças dos escores eram em média, razoavelmente, próximas nos dois períodos de tempo após o trauma, considerando a variabilidade dos dados.

Tabela 4 - Estatísticas descritivas das diferenças entre os escores dos comportamentos, após e antes do trauma, segundo o tempo do trauma. IC-HCFMUSP, 2001

\begin{tabular}{lcccc}
\hline \multicolumn{1}{c}{ COMPORTAMENTOS } & \multicolumn{4}{c}{ TEMPO } \\
& $\begin{array}{c}\text { Menor ou Igual a } 3 \\
\text { Média }\end{array}$ & Desvio Padrão & Média & Desvio Padrão 3 anos \\
\hline Agressividade & 1,97 & 2,01 & 2,25 & 1,62 \\
Ansiedade & 2,40 & 1,59 & 1,95 & 1,47 \\
Dependência & 2,37 & 1,71 & 2,15 & 1,76 \\
Depressão & 2,07 & 1,51 & 2,30 & 1,53 \\
Egocentricidade & 1,33 & 1,71 & 1,20 & 1,67 \\
Impulsividade & 1,50 & 1,78 & 1,85 & 1,57 \\
Inadequação social & 0,83 & 1,46 & 1,65 & 1,79 \\
Irritabilidade & 2,13 & 1,72 & 2,30 & 1,66 \\
Esquecimento & 2,53 & 1,46 & 2,30 & 1,56 \\
Oscilação do humor & 1,80 & 1,49 & 1,55 & 1,57 \\
Temperamento explosivo & 1,83 & 1,84 & 2,40 & 1,79 \\
\hline
\end{tabular}

Pôde-se concluir, a partir do teste de Mann Whitney, que as médias das diferenças dos escores atribuídos às vítimas com tempo de trauma menor ou igual a três anos eram iguais às médias das diferenças das vítimas com tempo do trauma maior que três anos, ou seja, não houve associação entre o tempo decorrido do trauma das vítimas e as mudanças nos escores de cada um dos comportamentos em estudo.

\section{DISCUSSÃO DOS RESULTADOS}

No presente estudo, os resultados relacionados a quanto os comportamentos das vítimas afetam o cuidador demonstraram claramente a tendência de aumento na intensidade para todos os comportamentos no período de seis meses ou mais após o trauma, em relação ao tempo anterior a esse evento. Entretanto, houve poucos casos de diminuição da intensidade que foram observados em relação à agressividade, ansiedade, depressão, impulsividade, irritabilidade, temperamento explosivo e dependência.

O esquecimento, comportamento que apresentou, em média a maior alteração, é um distúrbio da memória freqüente nas vítimas de $T C E$, e constitui a principal queixa cognitiva relativa às vítimas e suas famílias, dificultando o estabelecimento de nova aprendizagem ${ }^{(6)}$. A modificação do estado da memória dificulta a aprendizagem, pois são fenômenos interdependentes ${ }^{(14)}$.

Os cuidadores, durante as entrevistas, informaram que a vítima estava esquecida em muitos aspectos: esquecia de tomar 0 medicamento, de alimentar-se, tomar banho, do produto que ia comprar no supermercado, do dia da consulta e até mesmo quais eram os membros que pertenciam à sua família.

Além do esquecimento, o temperamento explosivo, agressividade, depressão, irritabilidade, ansiedade e dependência apresentaram maior alteração após o trauma, afetando mais o cuidador que os demais comportamentos das vítimas.

Observa-se na literatura diversidade de achados quanto aos comportamentos que mais afetam o cuidador pós-trauma, entre eles destaca-se: ansiedade, dependência e memória ${ }^{(12)}$; impaciência, impulsividade e infantilidade ${ }^{(15)}$; distúrbio do humor, labilidade, ansiedade, depressão, raiva, irritabilidade e agressão ${ }^{(8)}$; impaciência e depressão( ${ }^{(9)}$

Autores de uma das primeiras publicações sobre as conseqüências do TCE para a família concluíram que os efeitos negativos do TCE grave em cinco anos eram significativamente mais altos que em um ano ${ }^{(7)}$.

No atual estudo, contrapondo-se a esse resultado, não houve associação entre o tempo do trauma das vítimas e as mudanças na intensidade de cada comportamento, no entanto, mesmo após longos períodos, os efeitos comportamentais das vítimas de TCE continuavam causando impacto na vida dos cuidadores. Nesse sentido, um estudo ${ }^{(16)}$ 
com vítimas e familiares, realizado cerca de dez anos após o trauma, revelou que aproximadamente $80 \%$ dos membros da família apresentavam moderado a grave estresse, confirmando o impacto do TCE a longo prazo em suas vidas.

\section{CONCLUSÕES}

Quanto às diferenças observadas na Escala Likert em relação à intensidade que os comportamentos das vítimas afetavam o cuidador antes do trauma e o afetam atualmente ( $\geq$ seis meses após o trauma), pode-se concluir:

- as diferenças nos escores foram nulas, positivas e negativas. Nos comportamentos, egocentricidade, inadequação social, esquecimento e oscilação do humor, houve sempre manutenção ou aumento dos escores no período após o trauma; já nos comportamentos, agressividade, ansiedade, depressão, impulsividade, irritabilidade, temperamento explosivo e dependência, além da manutenção e aumento dos escores houve alguns casos de diminuição;

- houve aumento significativo nas médias dos escores de todos os comportamentos estudados seis meses ou mais após o trauma, em relação ao tempo anterior ao evento traumático, ou seja, na visão dos cuidadores as vítimas de TCE estavam mais agressivas, ansiosas, dependentes, deprimidas, egocêntricas, impulsivas, irritadas, esquecidas, com maior inadequação social, oscilação de humor e temperamento mais explosivo após o trauma;

\section{REFERÊNCIAS BIBLIOGRÁFICAS}

1. Ministério da Saúde (BR). Secretaria de Políticas de Saúde. Política nacional de redução da morbimortalidade por acidentes e violência. Rev Saúde Pública 2000; 34(4):427-30.

2. Nitrini R, Bacheschi LA. A neurología que todo médico debe saber. São Paulo (SP): Atheneu; 2003.

3. Souza CAC. Neuropsiquiatria dos traumatismos craniencefálicos. Rio de Janeiro (RJ): Revinter; 2003.

4. Sousa RMC, Regis FC, Koizumi MS. Traumatismo crânio-encefálico: diferenças das vítimas pedestres e ocupantes de veículos a motor. Rev Saúde Pública 1999; 33(1):85-94.

5. Leathem J, Heath E, Woolley C. Relatives'perceptions of role change, social support and stress after traumatic brain injury. Brain Inj 1996; 10(1):27-38.

6. Muñoz-Céspedes JM, Paúl-Lapedriza N, Pelegrín-Valero C, TirapuUstarroz J. Factores de pronóstico en los traumatismos craneoencefálicos. Rev Neurol 2001; 32(4):351-64.

7. Brooks N, Campsie L, Symington C, Beattie A, Mckinlay W. The five year outcome of severe blunt head injury: a relative's view. J Neurol Neurosurg Psychiatry 1986; 49(7):764-70.

8. Knight RTG, Devereux R, Godfrey H. Caring for a family member with a traumatic brain injury. Brain Inj 1998; 12(6):467-81.
- os comportamentos temperamento explosivo, agressividade, depressão, irritabilidade, ansiedade, dependência e esquecimento apresentaram maior alteração após o trauma, afetando mais o cuidador que os comportamentos inadequação social, egocentricidade, impulsividade e oscilação do humor;

- em média, o comportamento mais alterado das vítimas, segundo a percepção do cuidador foi o esquecimento.

Quanto à associação entre tempo do trauma e a diferença na Escala Likert:

- não houve associação entre as variáveis, portanto, as mudanças na intensidade que os comportamentos afetavam o cuidador não parecem estar relacionadas com o tempo do trauma da vítima.

\section{CONSIDERAÇÕES FINAIS}

Embora se observe no cenário internacional crescente número de pesquisas relativas aos efeitos a longo prazo do TCE na vida do cuidador, em âmbito nacional, nada até então havia sido pesquisado com essa especificidade.

Os efeitos das alterações comportamentais das vítimas de TCE para o cuidador familiar como se pôde constatar são adversos e persistentes também em nosso meio, e necessitam continuar sendo estudados com amostras diferenciadas de cuidadores e vítimas para que se possa aprimorar a assistência de enfermagem e oferecer melhor suporte social a essas pessoas.

9. Marsh NV, Kersel DA, Havill JH, Sleigh JW. Caregiver burden at 6 months following severe traumatic brain injury. Brain Inj 1998 ; 12(12):1045-59.

10. Choi SC, Barnes TY. Predicting outcome in the head injury patient. In: Narayan RK, editor. Neurotrauma. New York: Mac Graw Hill; 1996. p. 779-92.

11. Sousa RMC, Koizumi MS. Recuperação das vítimas de trauma craniencefálico entre 6 meses e 1 ano. Arq Bras Neurocir 1998; 17(2):72-80.

12. Frosch S, Gruber A, Jones C, Myers S, Noel E, Westerlund A. The long term effect of traumatic brain injury on the roles of caregivers. Brain Inj 1997; 11(12):891-906.

13. Hora ECH. Conseqüências do trauma crânio-encefálico para o cuidador familiar. [Dissertação]. São Paulo (SP): Escola de Enfermagem/USP; 2002.

14. Aguado-Aguilar L. Aprendizaje y memoria. Rev Neurol 2001; 32(4):373-81.

15. Marsh NV, Kersel DA, Havill JH, Sleigh JW. Caregiver burden at 1 year following severe traumatic brain injury. Brain Inj 1998; 12(12):104559.

16. Brzuzy S, Speziale BA. Persons with traumatic brain injuries and their families: living arrangements and well-being post injury. Soc Work Health Care 1997; 26(1):7-89. 